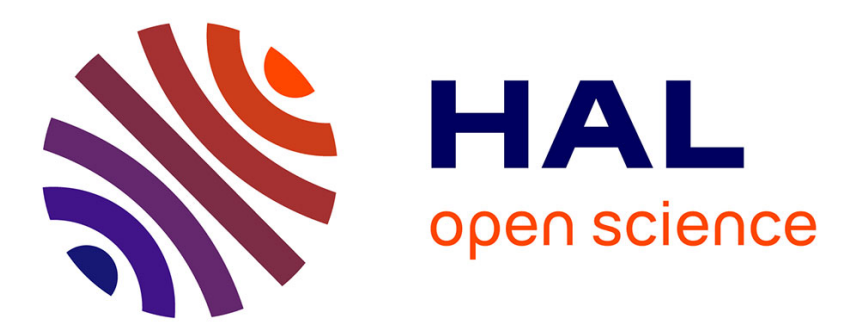

\title{
Chronique de jurisprudence de la Cour interaméricaine des droits de l'homme 2019
}

\author{
Marie Rota
}

\section{To cite this version:}

Marie Rota. Chronique de jurisprudence de la Cour interaméricaine des droits de l'homme 2019.

Cahiers de la recherche sur les droits fondamentaux , 2020, 10.4000/crdf.6487 . hal-03021725

\section{HAL Id: hal-03021725 \\ https://hal.science/hal-03021725}

Submitted on 24 Nov 2020

HAL is a multi-disciplinary open access archive for the deposit and dissemination of scientific research documents, whether they are published or not. The documents may come from teaching and research institutions in France or abroad, or from public or private research centers.
L'archive ouverte pluridisciplinaire HAL, est destinée au dépôt et à la diffusion de documents scientifiques de niveau recherche, publiés ou non, émanant des établissements d'enseignement et de recherche français ou étrangers, des laboratoires publics ou privés. 


\title{
Chronique de jurisprudence de la Cour interaméricaine des droits de l'homme 2019
}

\author{
Marie ROTA \\ Maître de conférences en droit public à l'université de Lorraine \\ Institut de recherches sur l'évolution de la nation et de l'État (IRENEE, EA 7303)
}

I. Le droit à la vie (article 4 de la CADH)
A. Les violations du droit à la vie imputables aux agents de l'État
B. La peine de mort: une exception strictement limitée
C. Le droit de mener une vie digne

II. Le droit à l'intégrité de la personne (article 5 de la CADH)
A. Le droit à l'intégrité et la peine de mort
B. Le droit à l'intégrité personnelle, à la dignité et à la sécurité sociale
C. L'intégrité de la personne et l'obligation procédurale d'enquête
D. Le droit à l'intégrité personnelle des personnes détenues

III. Le droit à la liberté de la personne (article 7 de la CADH)

IV. Les droits économiques, sociaux, culturels et environnementaux (article 26 de la CADH)

En 2008, lorsque la première «Chronique de jurisprudence de la Cour interaméricaine» a vu le jour dans ces Cahiers, la production scientifique en langue française y étant relative était assez rare. L'accès aux décisions de la Cour était difficile en raison de leur publication tardive sur son site Internet (encore accentuée s'agissant des traductions en langue anglaise par ailleurs de très mauvaise qualité). Beaucoup d'amalgames étaient faits s'agissant de cette juridiction présentée comme "petite sœur» de la Cour européenne ${ }^{1}$. C'est la raison pour laquelle nous avions décidé de publier cette chronique, à un rythme initialement annuel, permettant d'insister sur la spécificité de l'interprétation des droits humains effectuée par le juge de San José. Suite à la publication à partir de 2010 de ses rapports annuels présentant ses principales avancées jurisprudentielles en langue française ${ }^{2}$, un rythme bisannuel avait été retenu. L'apparition toujours plus fréquente de contributions consacrées à la jurisprudence de la Cour interaméricaine dans le monde académique francophone avait enfin motivé un abandon de cette chronique sur lequel nous revenons désormais. Car, si ces publications existent, elles ne sont que ponctuelles et ne concernent que certains sujets donnés ${ }^{3}$. Les rapports annuels de la Cour ne paraissent en outre que tardivement. Or ses avancées jurisprudentielles sont dorénavant scrutées tant par la Cour européenne que par le monde académique et la société

1. M. Rota, "Chronique de jurisprudence de la Cour interaméricaine des droits de l'homme », Cahiers de la recherche sur les droits fondamentaux, $\mathrm{n}^{\circ} 6,2008$, p. 181-182.

2. Ces rapports sont disponibles sur la page suivante: http://www.corteidh.or.cr/informe-anual.cfm.

3. Exception faite de la très belle chronique de jurisprudence publiée par Hélène Tigroudja portant néanmoins sur une période de trois années (H. Tigroudja, "Chronique de la jurisprudence consultative et contentieuse de la Cour interaméricaine des droits de l'homme (2015-2017)", Revue trimestrielle des droits de l'homme, $\mathrm{n}^{\circ}$ 115, 2018, p. 685-728). 
civile, qui trouvent ici un point d'appui pour faire « avancer le droit ${ }^{4}$ dans un sens, on l'espère, plus protecteur des droits humains. C'est pourquoi une analyse pointue et relativement exhaustive ${ }^{5}$ de cette jurisprudence sur l'année passée nous apparaît aujourd'hui pertinente.

S'agissant du contentieux, on observe une nette diversification en 2019 qui s'accompagne d'un certain judicial self-restraint. Une seule affaire a trait aux disparitions forcées, dans laquelle la Cour constate cependant l'absence de contexte systématique et généralisé y étant relatif. Il en découle une inversion de la charge de la preuve, reposant sur les requérants, qui ne sont pas parvenus à démontrer que les victimes étaient détenues par des agents de l'État ${ }^{6}$, ainsi qu'une disparition de l'obligation positive d'enquête ex officio ${ }^{7}$. L'affaire Rico $c$. Argentine est aussi assez révélatrice de la retenue de la Cour, car, si elle refuse de manière traditionnelle de se référer à la marge nationale d'appréciation, elle se livre en l'espèce à un contrôle de l'erreur manifeste d'appréciation s'agissant des décisions rendues par les juridictions internes ${ }^{8}$. On peut enfin citer l'affaire Virula et autres c. Guatemala, relative à l'assassinat d'un syndicaliste dans laquelle la Cour refuse de reconnaître l'existence d'une obligation de prévention qui serait à la charge de l'État au regard des risques d'atteinte aux droits à la vie, à la liberté personnelle, à l'intégrité personnelle et à la liberté d'association de la victime, au motif qu'il n'est pas démontré que l'État savait ou aurait dû avoir connaissance de la «situation de risque avéré et imminent» dans laquelle se trouvaient les membres du syndicat, avant la disparition de la victime ${ }^{9}$. On prend ici conscience de l'importance de la charge de la preuve, élément crucial pour l'issue d'une affaire ${ }^{10}$.

Faute d'espace, nous laisserons de côté les décisions qui ne font que reprendre des principes bien ancrés dans la jurisprudence de la Cour, à savoir l'affaire Álvarez Ramos c. Venezuela relative à la liberté d'expression, aux droits politiques et à la liberté d'aller et venir d'un journaliste condamné pour diffamation ${ }^{11}$, et les affaires Colindres Schonenberg c. El Salvador ${ }^{12}$, Gorigoitía c. Argentine ${ }^{13}$ et Rosadio Villavicencio c. Pérou ${ }^{14}$, qui ont essentiellement trait aux articles 8 et 25 de la Convention américaine des droits de l'homme (CADH) protégeant les droits aux garanties et à la protection judiciaires. Bien qu'il s'agisse des dispositions les plus invoquées par les requérants, y compris dans les autres affaires, et qu'elles présentent un réel intérêt notamment au regard des relations entretenues entre la Cour et les juridictions nationales, nous nous attarderons, dans le cadre de cette chronique, uniquement sur le droit à la vie (I), le droit à l'intégrité (II), le droit à la liberté de la personne (III) et les droits économiques, sociaux, culturels et environnementaux (IV).

\section{Le droit à la vie (article 4 de la CADH)}

La Cour précise la portée du droit à la vie dans plusieurs affaires. Elle rappelle que l'article 4 , lu conjointement avec l'article 1.1 qui impose aux États de respecter les droits et libertés conventionnels, ne suppose pas uniquement une abstention de leur part - et donc "pas seulement que nulle personne soit privée de sa vie de manière arbitraire (obligation négative)»- mais implique aussi qu'ils doivent adopter

[...] toutes les mesures appropriées pour protéger et préserver le droit à la vie (obligation positive), conformément au devoir de garantir le plein et libre exercice des droits à toute personne relevant de leur juridiction ${ }^{15}$.

Au titre de la première obligation, on retrouve l'interdiction faite aux agents de l'État de violer ce droit (A) mais aussi d'infliger la peine de mort en dehors des conditions prévues par la Convention (B). Au titre de la seconde, on trouve le droit de mener une vie digne, qui marque la spécificité de la jurisprudence interaméricaine $(\mathrm{C})$.

4. Cette expression est empruntée à Françoise Tulkens, témoignage vidéo relatif à l'avis juridique rendu par le Tribunal international Monsanto, en ligne: https://vimeo.com/213865446 (13 ${ }^{\mathrm{e}}$ seconde).

5. Faute d'espace, seules les décisions portant sur le fond des affaires soumises à l'examen de la Cour feront l'objet d'une analyse ainsi que ses opinions consultatives. De la même façon, seule l'interprétation de la première partie de la Convention, intitulée «Des obligations des États et des droits protégés", sera abordée.

6. La Cour ne parle que d'indices présentés devant elle, non corroborés par de véritables preuves qui auraient été apportées en plus de ce qu'a étudié l'État dans le cadre des procédures internes (Cour IDH, Arrom Suhurt et autres c. Paraguay, fond, 13 mai 2019, série C, $\mathrm{n}^{\circ} 377, \$ 96$ ).

7. Cour IDH, Arrom Suhurt et autres c. Paraguay, $\$ 138-141$.

8. S'agissant de l'appréciation des éléments de preuves et de leur rejet par les juridictions internes, la Cour estime, tout comme la Commission par ailleurs, que "cette décision judiciaire doit être fondée sur des motifs "manifestement déraisonnables ou incompatibles avec les normes applicables" pour constituer une violation des garanties judiciaires» (Cour IDH, Rico c. Argentine, exception préliminaire et fond, 2 septembre 2019, série C, $\left.n^{\circ} 383, \$ 82\right)$. Il en va de même s'agissant des décisions internes rejetant certaines voies de recours jugées comme étant inappropriées, qui ne sont, selon la Cour, "ni manifestement arbitraires ni déraisonnables et donc contraires à la Convention américaine» (ibid., \$ 99). L'auteure précise ici que, sauf mention contraire, les traductions sont les siennes.

9. Cour IDH, Virula et autres c. Guatemala, exception préliminaire, fond, réparations et frais, 21 novembre 2019 , série C, $\mathrm{n}^{\circ} 393, \$ 55-60$.

10. Voir sur ce point le projet de recherche porté par Marie-Bénédicte Dembour intitulé «DISSECT: Evidence in International Human Rights Adjudication", dont l'argumentaire est disponible en ligne: https://hrc.ugent.be/wp-content/uploads/2019/10/DISSECT_description.pdf.

11. Cour IDH, Álvarez Ramos c. Venezuela, exception préliminaire, fond, réparations et frais, 30 août 2019, série C, $\mathrm{n}^{\circ} 380$. La Cour rappelle en l'espèce son test de compatibilité avec la Convention des restrictions aux trois droits invoqués, tout en insistant sur la vision large de la liberté d'expression dont elle a toujours fait la promotion, particulièrement au regard de propos d'intérêt général.

12. Cour IDH, Colindres Schonenberg c. El Salvador, fond, réparations et frais, 4 février 2019, série C, $n^{\circ} 373$.

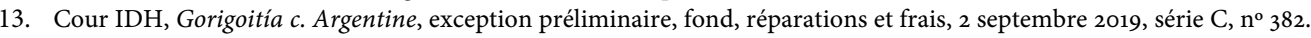

14. Cour IDH, Rosadio Villavicencio c. Pérou, exceptions préliminaires, fond, réparations et frais, 14 octobre 2019 , série $\mathrm{C}$, $\mathrm{n}^{\circ} 388$.

15. Cette affirmation de principe est réitérée de manière systématique dans les différentes affaires traitant du droit à la vie soumises à notre étude. Voir, par exemple, Cour IDH, Ruiz Fuentes et autre c. Guatemala, exception préliminaire, fond, réparations et frais, 10 octobre 2019 , série C, $\mathrm{n}^{\circ} 384, \$ 100$. 


\section{A. Les violations du droit à la vie imputables aux agents de l'État}

En vertu du droit à la vie, les États ont tout d'abord l'obligation de garantir les conditions requises pour que ce «droit inaliénable» ne soit pas violé et, au premier chef, d'empêcher ses agents d'y porter atteinte ${ }^{16}$. Cela concerne non seulement le pouvoir législatif, mais aussi «toute institution étatique», y compris les forces armées et de police $^{17}$. Ce sont justement ces dernières qui sont mises en cause dans plusieurs affaires.

Dans la première, Díaz Loreto et autres $c$. Venezuela ${ }^{18}$, la Cour se penche sur l'usage de la force (coups de feu) par des agents de l'État ayant abouti au décès des victimes. Elle rappelle sa jurisprudence classique en affirmant que, pour être compatible avec la Convention, l'usage de la force doit être prévu par la loi, poursuivre un but légitime, être nécessaire et strictement proportionné ${ }^{19}$. Elle affirme ensuite l'existence d'un contexte d'exécutions extrajudiciaires commises par des agents de la police vénézuélienne, reconnu par les institutions publiques tant au niveau interne

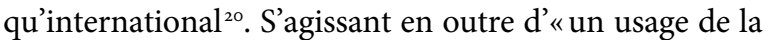
force par des agents de l'État ayant occasionné des blessures ou la mort à une ou plusieurs personnes", le Venezuela a l'obligation de «fournir une explication satisfaisante et convaincante sur ce qui s'est passé et de désavouer les allégations relatives à sa responsabilité, au moyen d'éléments de preuve adéquats ${ }^{21}$. Il en résulte une inversion de la charge de la preuve, et la Cour, se focalisant sur les deux derniers critères de son test de compatibilité, constate que l'État n'a pas prouvé qu'il y avait eu une confrontation entre les victimes et les forces de police ${ }^{22}$. Il en découle une violation du droit à la vie des victimes, sans pour autant qu'elle ne soit qualifiée d'exécution extrajudiciaire ${ }^{23}$.

Les forces de police sont également mises en cause dans l'affaire Ruiz Fuentes et autre c. Guatemala, relative au décès d'un fugitif condamné à la peine de mort pour avoir enlevé un enfant et demandé en rançon le paiement d'un million de quetzales à son père. Après avoir été placé dans un centre de détention de sécurité maximale dénommé El Infiernito, il s'en évade avec dix-huit autres détenus. En vue de retrouver les fugitifs, l'État lance une opération policière de grande ampleur, baptisée Gavilán. Le corps de la victime est retrouvé un mois plus tard criblé de balles. La Cour retient la responsabilité des agents de l'État au regard d'un ensemble de données. Un rapport d'expert indique tout d'abord que le tir de la balle retrouvée dans ses yeux avait eu lieu à courte distance, ce qui rend difficilement crédible la version des faits présentée par l'État selon laquelle la victime avait trouvé la mort suite à un affrontement armé ${ }^{24}$. Il est ensuite prouvé que les membres du Comando Antisecuestros de la police nationale étaient sur le lieu du crime et que sa scène a été altérée $^{25}$. Le Guatemala a en outre fait état de plusieurs versions contradictoires au niveau interne, devant la Commission et devant la Cour ${ }^{26}$. Il ressort enfin de la version des faits présentée par le ministère public chargé de l'enquête pénale ouverte après la mort du requérant qu'il avait été victime d'une exécution extrajudiciaire, ce à quoi conclut aussi la Cour ${ }^{27}$.

\section{B. La peine de mort: une exception strictement limitée}

La Cour a eu à se pencher sur la compatibilité de la peine de mort prononcée à l'encontre de plusieurs personnes au Guatemala avec l'article 4 de la Convention dans quatre affaires. Elle se livre dans ce cadre à une interprétation à la fois textuelle, systématique et consensuelle de cette disposition pour signaler la «tendance abolitionniste» de cette peine, qui implique qu'elle ne soit acceptable que dans des cas exceptionnels et soumise à de strictes limitations ${ }^{28}$. Ceci ressort tant de la formulation de l'article 4 et plus particulièrement de ses alinéas deux à six ${ }^{29}$, de sa jurisprudence consultative ${ }^{30}$, du Protocole additionnel à la CADH relatif à l'abolition de la peine de mort $^{31}$, que du système universel de protection des droits humains avec lequel le système interaméricain est donc «en harmonie $»^{32}$. La Cour rappelle enfin son affaire Fermín Ramírez c. Guatemala ${ }^{33}$

16. Cour IDH, Ruiz Fuentes et autre c. Guatemala, $\$ 100$.

17. Ibid.

18. Cour IDH, Díaz Loreto et autres c. Venezuela, exceptions préliminaires, fond, réparations et frais, 19 novembre 2019 , série C, $\mathrm{n}^{\circ} 392$.

19. Ibid., $\$ 63$.

20. Ibid., $\$ 66-68$

21. Ibid., $\$ 88$.

22. Ibid., $\$ 87$.

23. Cet aspect est critiqué par le président de la Cour Eduardo Ferrer Mac-Gregor Poisot et la juge Elizabeth Odio Benito dans leur opinion dissidente.

24. Cour IDH, Ruiz Fuentes et autre c. Guatemala, $\$ 103$.

25. Ibid., \$104-106.

26. Ibid., $\$ 107-109$

27. Ibid., $\$ 110-111$.

28. Cour IDH, Martínez Coronado c. Guatemala, fond, réparations et frais, 10 mai 2019, série C, $n^{\circ} 376, \$ 62$.

29. Ibid., $\$ 62-63$.

30. Cour IDH, Restricciones a la pena de muerte (arts. 4.2 y 4.4 Convención Americana sobre Derechos Humanos), opinion consultative n ${ }^{\circ}$ OC-3/83, 8 septembre 1983 , série $A, n^{\circ} 3, \$ 53$.

31. Cour IDH, Martínez Coronado c. Guatemala, $\$ 64-66$. Protocole à la Convention américaine relative aux droits de l'homme traitant de l'abolition de la peine de mort, adopté à Asunción (Paraguay), le 8 juin 1990, en ligne: https://www.cidh.oas.org/Basicos/French/g.peinedemort.htm.

32. Cour IDH, Martínez Coronado c. Guatemala, $\$ 67$.

33. Cour IDH, Fermín Ramírez c. Guatemala, fond, réparations et frais, 20 juin 2005, série C, n $126, \S 96$ et 98 . 
dans laquelle elle s'était déjà prononcée sur le concept de «dangerosité future » d'un détenu en signalant que l'utilisation de ce critère tant au niveau de la qualification juridique des faits qu'au niveau de la détermination de la sanction applicable est incompatible avec le principe de légalité (article 9 de la CADH). En effet elle « suppose une sanction fondée sur un jugement de la personnalité du délinquant et pas sur les infractions imputées en vertu de la classification pénale applicable ${ }^{34}$. Elle en déduit une violation de cette disposition tout comme celle des articles 4.1 et article 4.2 en soulignant que cette peine a, à la différence de l'affaire précitée ${ }^{35}$, été cette fois-ci exécutée ${ }^{36}$. C'est pourtant bien à un revirement de jurisprudence qu'elle procède puisque quelques mois plus tard, dans deux autres décisions, elle accepte d'évaluer cette peine au regard du droit à la vie et constate une violation de l'article 4.2 relatif au champ d'application de la peine de mort $^{37}$ alors même qu'elle n'a pas été exécutée ${ }^{38}$.

Elle estime que cette même disposition est par ailleurs violée dans l'affaire Ruiz Fuentes et autres c. Guatemala s'agissant cette fois-ci de la condamnation d'un détenu à la peine de mort en raison du crime commis, l'enlèvement. Cette affaire s'inscrit ici dans la continuité de sa jurisprudence, la loi pénale guatémaltèque relative à la peine de mort ayant déjà été condamnée par la Cour dans une affaire précédente ${ }^{39}$. Elle constate, de la même façon, une extension du champ d'application de cette peine ${ }^{40}$, qui s'accompagne d'un caractère automatique et obligatoire, incompatible avec l'article $4.2^{41}$. Enfin, l'article 4.6 est violé en raison de la suppression de la procédure de demande de grâce organisée devant un organisme étatique spécialement constitué pour ce faire ${ }^{42}$.

\section{Le droit de mener une vie digne}

La reconnaissance du droit de mener une vie digne est une spécificité de la jurisprudence de la Cour interaméricaine, lorsqu'on la compare, tout du moins, avec celle de sa consœur européenne. Contrairement à cette dernière ${ }^{43}$, elle considère - ce qu'elle rappelle dans l'affaire ANCEJUBSUNAT c. Pérou - que ce droit doit non seulement se comprendre comme celui de «tout être humain à ne pas être privé de la vie de manière arbitraire, mais aussi comme le droit à ne pas se voir imposer des conditions qui l'empêchent ou compliquent l'accès à une existence digne ${ }^{4}$. La Cour interaméricaine accepte donc d'instituer une protection contre la violence sociale et de donner un contenu concret au terme de «vie» en invoquant la dignité humaine. Elle implique que l'État s'assure que chacun ait accès à des conditions de vie minimales, dignes d'un être humain et qui n'aboutissent pas à sa réification. L'État a par conséquent l'obligation «d'adopter des mesures positives, concrètes et visant à satisfaire le droit à une vie digne, en particulier lorsqu'il s'agit de personnes en situation de vulnérabilité et de risque ${ }^{45}$. Dans cette hypothèse, l'attention que l'État doit porter à ces personnes «devient prioritaire ${ }^{46}$. C'est le cas, comme en l'espèce, des personnes âgées ${ }^{47}$.

La Cour accepte par ailleurs de faire le lien entre ce droit et celui qu'elle a découvert dans une affaire précédant l'affaire ANCEJUB-SUNAT c. Pérou de quelques mois, à savoir le droit à la sécurité sociale ${ }^{48}$, dont l'un des composants est «la retraite reposant sur un système de contributions ou de cotisations [sociales] ${ }^{49}$. Soulignant le fait qu'elle « constitue le seul salaire de remplacement

34. Cour IDH, Martínez Coronado c. Guatemala, $\$ 70$. Voir aussi Cour IDH, Fermín Ramírez c. Guatemala, $\$ 94$.

35. Dans l'affaire Fermín Ramírez c. Guatemala, $\$ 103$, elle avait en effet considéré que «si [la victime] avait été exécutée en raison de la procédure menée à son encontre, une privation arbitraire du droit à la vie aurait été caractérisée au regard de l'article 4 de la Convention".

36. Cour IDH, Martínez Coronado c. Guatemala, $\$ 71$. Dans cette affaire, la victime avait été condamnée à la peine de mort en raison de sa dangerosité après avoir été reconnue coupable d'un assassinat de sept personnes. Elle a fait l'objet d'une injection létale après plusieurs recours intentés dans l'ordre juridique interne.

37. Selon cette disposition, «Dans les pays qui n’ont pas aboli la peine de mort, celle-ci ne pourra être infligée qu'en punition des crimes les plus graves en vertu d'un jugement définitif rendu par un tribunal compétent en application d'une loi prévoyant cette peine qui était en vigueur avant la perpétration du crime. La peine de mort ne sera pas non plus appliquée à des crimes qu'elle ne sanctionne pas actuellement».

38. Cour IDH, Valenzuela Ávila c. Guatemala, fond, réparations et frais, 11 octobre 2019, série C, no $386, \$ 156$ et Cour IDH, Rodríguez Revolorio et autres $c$. Guatemala, exception préliminaire, fond, réparations et frais, 14 octobre 2019 , série $\mathrm{C}, \mathrm{n}^{\circ} 387, \S 64$. Il n’y a donc violation du premier alinéa de cette disposition, qui interdit les exécutions arbitraires, que si cette peine a été effectivement appliquée (Cour IDH, Valenzuela Ávila c. Guatemala, $\$ 156$ et Cour IDH, Rodríguez Revolorio et autres c. Guatemala, $\$ 65)$.

39. Cour IDH, Raxcacó Reyes c. Guatemala, fond, réparations et frais, 15 septembre 2005, série C, nº 133.

40. Cour IDH, Ruiz Fuentes et autre c. Guatemala, $\$ 85-86$.

41. En effet, ni les circonstances particulières de l'espèce, ni «le degré de participation et de culpabilité de l'accusé» ne peuvent, de ce fait, être invoqués, circonstances qui pourraient être de nature à atténuer la sanction prononcée. Or, dans la mesure où l'article 4.2 restreint l'imposition de la peine de mort aux crimes les plus graves, cette disposition est enfreinte (Cour IDH, Ruiz Fuentes et autre c. Guatemala, $\$ 88$ ). La Cour réitère ce point de vue dans l'affaire Cour IDH, Girón et autre c. Guatemala, exception préliminaire, fond, réparations et frais, 15 octobre 2019 , série C, $\mathrm{n}^{\circ} 390, \$ 71$.

42. Cour IDH, Ruiz Fuentes et autre c. Guatemala, $\$ 91$.

43. Selon la Cour $\mathrm{EDH}$, «l'article 2 assure uniquement une protection contre le fait d'infliger la mort» et «ne peut pas être interprété comme garantissant le droit à une certaine qualité de vie» (Cour EDH, $1^{\text {re }}$ section, 29 avril 2003, Dremlyuga c. Lettonie, $\mathrm{n}^{\circ}$ 66729/o1).

44. Cour IDH, Asociación Nacional de Cesantes y Jubilados de la Superintendencia Nacional de Administración Tributaria (ANCEJUB-SUNAT) c. Pérou, exceptions préliminaires, fond, réparations et frais, 21 novembre 2019 , série $C, \mathrm{n}^{\circ} 394, \$ 186$. Elle rappelle à ce titre sa jurisprudence classique selon laquelle la fondamentalité du droit à la vie, qui conditionne l'exercice des autres, implique qu'il soit aussi compris comme consacrant le droit pour toute personne de ne "pas être empêchée d'avoir accès à des conditions qui lui garantissent une existence digne " (ibid.).

45. Cour IDH, ANCEJUB-SUNAT c. Pérou, $\$ 186$.

46. Ibid.

47. Ibid.

48. Cour IDH, Muelle Flores c. Pérou, exceptions préliminaires, fond, réparations et frais, 6 mars 2019, série C, nº $375, \$ 187$ (voir infra).

49. Cour IDH, ANCEJUB-SUNAT c. Pérou, $\$ 184$. 
que [les personnes âgées] reçoivent pour subvenir à leurs besoins essentiels ", la Cour estime que la retraite, mais aussi la sécurité sociale d'une manière générale, «constituent un moyen de protection pour jouir d'une vie digne ${ }^{50}$. Aussi, le «droit à la sécurité sociale et le droit à la vie digne sont interconnectés, et de manière encore plus accentuée s'agissant de personnes âgées $»^{51}$. La Cour estime qu'il en découle plusieurs obligations positives à la charge de l'État en vue de protéger la dignité de la vie des personnes âgées, en se fondant sur le «corpus juris international» pertinent en la matière ${ }^{52}$. Sans pour autant les lister de manière précise, elle constate qu'en l'espèce la diminution du montant des retraites des victimes avait eu un impact direct sur «leurs besoins fondamentaux en termes de santé, de logement, de nourriture et d'éducation pour leurs enfants ${ }^{53}$. La qualité de vie des victimes s'en est trouvée affectée, ce qui implique «non seulement une violation de leur droit à la sécurité sociale, mais aussi une violation du droit à une vie digne $»^{54}$.

\section{Le droit à l'intégrité de la personne (article 5 de la CADH)}

Le droit à l'intégrité de la personne a connu des développements spécifiques tant au regard de la peine de mort (A), de sa lecture combinée avec le droit à la dignité et le droit à la sécurité sociale (B), de ses prolongements procéduraux $(\mathrm{C})$ que de son invocation à l'égard des personnes détenues (D).

\section{A. Le droit à l'intégrité et la peine de mort}

Dans les différentes affaires relatives à la peine de mort, la Cour réitère sa position classique selon laquelle le fait de se savoir dans le "couloir de la mort» porte atteinte non seulement à l'intégrité physique, psychique et morale protégée par l'article 5.1 de la Convention, mais constitue aussi un traitement cruel, inhumain et dégradant prohibé par l'article $5 \cdot 2^{55}$. De même, l'angoisse et la souffrance ressenties par les membres de la famille violent cette première disposition ${ }^{56}$. Dans l'affaire Girón et autre c. Guatemala, la Cour souligne aussi que le fait que l'exécution ait été retransmise à la télévision pour «donner l'exemple» porte atteinte à la dignité humaine puisque cela revient à traiter les victimes $"$ comme des objets ${ }^{57}$. Il s'agit donc d'un traitement dégradant ${ }^{58}$. Elle refuse en revanche de répondre à l'allégation de la Commission et des représentants des victimes selon laquelle le mode choisi pour mettre en œuvre cette exécution, à savoir le fait d'être fusillé, constituait ou non un acte de torture ${ }^{59}$, alors même qu'elle reconnaît que, tant au niveau régional qu'universel, les modes d'exécution de la peine capitale qui causent le plus de souffrance sont interdits ${ }^{60}$. Néanmoins, «en raison des violations déjà déclarées en l'espèce, la Cour estime qu'il n'est pas nécessaire de se prononcer sur [cette question] ${ }^{61}$.

\section{B. Le droit à l'intégrité personnelle, à la dignité et à la sécurité sociale}

La Cour se penche dans l'affaire Muelle Flores c. Pérou sur la réduction des pensions touchées par la victime du fait de son reclassement dans le régime de retraite de droit commun. Alors que les juridictions internes ont invalidé cette décision, la victime, ayant travaillé dans une mine d'État durant toute sa carrière et avant qu'elle ne soit privatisée, n'a jamais concrètement réintégré le régime des retraites des fonctionnaires. Après avoir conclu à la violation du droit à une retraite tel que protégé par l'article $26^{62}$, la Cour souligne que le droit à la sécurité sociale (dont il fait partie intégrante), le droit à l'intégrité personnelle et le droit à la dignité humaine sont "interdépendants», ce qui implique que «la violation de l'un puisse affecter directement l'autre», d'autant plus lorsqu'il s'agit de personnes âgées ${ }^{63}$. En effet, «le non-paiement des allocations de retraite génère chez une personne âgée une atteinte directe à sa dignité » puisqu'il s'agit de la principale ressource dont elle dispose "pour régler ses besoins primaires et élémentaires en tant qu'être humain ${ }^{64}$, ainsi qu'une "angoisse, insécurité et incertitude quant à [son] avenir", d'où une atteinte à son droit à l'intégrité ${ }^{65}$. Aussi, et même si les articles 5.1 et 11.1, qui protègent l'intégrité et la dignité de la personne, n'ont pas été expressément invoqués ni par la Commission ni par les représentants des victimes, la Cour estime opportun

50. Ibid.

51. Ibid., $\$ 185$

52. Ibid., $\$ 187$.

53. Ibid., $\$ 188$.

54. Ibid., $\$ 191$.

55. Cour IDH, Ruiz Fuentes et autre c. Guatemala, \$137; Cour IDH, Valenzuela Ávila c. Guatemala, \$207; Cour IDH, Rodríguez Revolorio et autres c. Guatemala, $\$ 96$.

56. Cour IDH, Ruiz Fuentes et autre c. Guatemala, $\$ 191$.

57. Cour IDH, Girón et autre c. Guatemala, $\$ 87$.

58. Ibid.

59. Ibid., $\$ 89$.

60. Ibid., $\$ 80$.

61. Ibid., $\$ 89$.

62. Voir infra.

63. Cour IDH, Muelle Flores c. Pérou, $\$ 204$.

64. Ibid., $\$ 205$.

65. Ibid., $\$ 206$. 
de s'en saisir en vertu «du principe général du Droit iura novit curia ${ }^{66}$. Ces considérations sont fondamentales puisqu'elles ont motivé l'invocation par les représentants des victimes du droit de mener une vie digne dans l'affaire ANCEJUB-SUNAT c. Pérou sus-analysée. En l'espèce, le fait d'avoir privé la victime, bénéficiant en outre d'une «protection spécifique en tant que personne âgée en situation de handicap", de son droit à la sécurité sociale pendant plus de vingt-sept ans a abouti à ce que sa "qualité de vie et sa couverture de santé» aient été gravement affectées ${ }^{67}$. Une situation de "précarité économique» en a découlé, d'où une «atteinte à son intégrité psychologique et morale ainsi qu'à sa dignité ${ }^{68}$.

\section{L'intégrité de la personne et l'obligation procédurale d'enquête}

Dans l'affaire Villaseñor Velarde et autres c. Guatemala, la Cour se prononce sur un contexte d'insécurité des acteurs de la justice entre 1990 et 2012, ces derniers étant victimes de menaces et d'actes intimidation récurrents, voire d'agressions liées à leurs fonctions, altérant l'indépendance judiciaire, sans que l'État ne tente d'y remédier. La requérante, juge entre 1990 et 2013, a dénoncé devant les juridictions internes plusieurs menaces directes, des dommages intentés à l'encontre de ses biens, des tentatives d'accéder à son domicile, entre autres. La Cour relève en l'espèce l'existence de graves tentatives d'intimidation ${ }^{69}$, dont l'État avait eu connaissance ${ }^{70}$. Elle rappelle sa jurisprudence antérieure selon laquelle, en vue d'éviter que l'indépendance judiciaire ne soit affectée par des "pressions externes ", l'État doit prévenir de telles ingérences, mener une enquête et sanctionner les responsables. Or, ce fut bien le cas s'agissant de la première obligation au regard de la mise à disposition de la victime de forces de sécurité pendant près de dix-huit ans ${ }^{71}$. L'obligation procédurale d'enquête a en revanche été malmenée ${ }^{72}$. La Cour insiste à ce titre sur le fait qu'elle ne vise pas seulement à satisfaire les exigences découlant des articles 8 et 25 de la Convention qui protègent le droit aux garanties et à une protection judiciaires, mais a aussi vocation à permettre «la jouissance [des] droits substantiels [de la requérante] et sa qualité de juge ${ }^{73}$. Il en va aussi de l'exigence d'indépendance de la justice ${ }^{74}$. Au regard des différents éléments de preuve soumis à la Cour, cette dernière estime que les défaillances de l'État en la matière ont abouti à ce que l'intégrité personnelle de la requérante, en tant que juge, ait été violée ${ }^{75}$. L'État est par conséquent responsable d'une violation des articles 5.1, 8.1 et 25.1 de la Convention, lus en relation avec son article 1.1.

\section{Le droit à l'intégrité personnelle des personnes détenues}

La Cour réitère sa jurisprudence classique s'agissant de la qualification d'actes de torture commis par les agents de l'État à l'encontre de détenus dans trois affaires. Les deux premières (Ruiz Fuentes et autre c. Guatemala et Valenzuela Ávila c. Guatemala) sont relatives à la peine de mort. La Cour y rappelle que le mauvais traitement doit, pour être qualifié de torture, être intentionnel, causer de graves souffrances physiques et mentales et être finalisé ${ }^{76}$. C'est bien le cas dans la première affaire au regard du caractère intentionnel des traitements subis par la victime (sévices physiques graves), des souffrances physiques éprouvées et de la finalité des tortionnaires (les forces de police) qui était d'obtenir des informations sur d'autres enlèvements ${ }^{77}$. Il en va de même dans la seconde, les mauvais traitements occasionnés (coups, asphyxie et pénétration anale au moyen d'un bâton) ayant cette fois-ci pour fin d'obtenir des aveux ${ }^{78}$ ou des informations sur le décès de la personne assassinée par la victime ${ }^{79}$. La Cour réaffirme à cette occasion sa jurisprudence en matière de viol en signalant que «les souffrances graves de la victime sont inhérentes au viol et qu'un viol peut constituer une torture même s'il consiste en un seul acte ou se produit en dehors des locaux de l'État ${ }^{80}$.

L'affaire Díaz Loreto et autres c. Venezuela permet ensuite à la Cour de rappeler qu'est aussi qualifié d'acte de torture tout usage de la force par les agents de l'État qui n'est pas strictement rendu nécessaire par le comportement du détenu, en ce qu'il constitue « une atteinte à la dignité humaine ${ }^{81}$. Elle réaffirme par ailleurs que

66. Cour IDH, Muelle Flores c. Pérou, $\$ 204$.

67. Ibid., $\$ 207$.

68. Ibid.

69. Cour IDH, Villaseñor Velarde et autres c. Guatemala, fond, réparations et frais, 5 février 2019, série C, $\mathrm{n}^{\circ} 374, \S 89$.

70. Ibid., $\$ 95$.

71. Ibid., $\$ 104$

72. Ibid., $\$ 126$.

73. Ibid., $\$ 130$

74. Ibid.

75. Ibid., $\$ 131$.

76. Selon la Cour, en effet, est qualifiée de torture «i) un acte intentionnel; ii. qui a provoqué de sévères souffrances physiques ou mentales, et iii) s'il a été commis avec un objectif ou une finalité déterminée» (Cour IDH, Ruiz Fuentes et autre c. Guatemala, \$129 et Cour IDH, Valenzuela Ávila c. Guatemala, \$193).

77. Cour IDH, Ruiz Fuentes et autre c. Guatemala, $\$ 130-131$.

78. Cour IDH, Valenzuela Ávila c. Guatemala, $\$ 195$.

79. Ibid., $\$ 201$.

80. Ibid., $\$ 194$.

81. Cour IDH, Díaz Loreto et autres c. Venezuela, $₫ 91$. 
la charge de la preuve revient dans ce cas à l'État et qu'il existe une présomption de responsabilité pour les blessures d'une personne ayant été «sous la garde des agents de l'État " ${ }^{82}$. N'ayant ni enquêté ni apporté d'explications satisfaisantes s'agissant de la mort d'un détenu, l'État est reconnu responsable d'une violation de son droit à l'intégrité tel que protégé par l'article 5.1 de la Convention.

S'agissant des conditions de détention en tant que telles, la Cour rappelle qu'elles peuvent être constitutives d'un traitement cruel, inhumain et dégradant ${ }^{8_{3}}$ lorsqu'elles ne remplissent pas les «exigences matérielles minimales d'un traitement digne $»^{84}$, comme c'est le cas au sein du centre de sécurité maximale El Infiernito au Guatemala ${ }^{85}$. Elle se livre par ailleurs à une lecture combinée des articles 5.1, 5.2 avec l'article 26 de la Convention qui protège les droits économiques, sociaux et culturels dans l'affaire Hernándezc. Argentine ${ }^{86}$. En l'espèce, suite à la contraction d'une méningite tuberculeuse durant sa détention, l'état de santé de la victime ne cesse de se dégrader (atteintes neurologiques impliquant la perte de la vision d'un œil, incapacité partielle et permanente du membre supérieur gauche, perte de mémoire notamment). La Cour estime que l'intégrité personnelle du détenu a été violée tant en raison de la surcharge du lieu de détention que de l'absence de prise en charge médicale suffisante ${ }^{87}$. Elle constate à ce titre l'existence d'omissions imputables à l'État en termes de qualité, de disponibilité et d'accessibilité en matière de soins. Il en ressort une violation du droit à l'intégrité de la victime et de son droit à la santé, justiciable en tant que tel ${ }^{88}$.

Dans l'affaire López et autres c. Argentine ${ }^{89}$, enfin, la Cour se prononce sur le transfert de détenus mineurs dans des centres de détention se situant à huit cent et deux mille kilomètres de distance de leur famille, de leurs avocats et des juges chargés de l'exécution de leur peine. La Cour se fonde sur l'article 5.6 de la Convention selon lequel « [1]es peines privatives de liberté doivent avoir pour but essentiel l'amendement et le reclassement social des condamnés» qu'elle lit en relation avec l'article $5.3^{90}$ imposant, selon elle, que les effets de la privation de liberté soient strictement rendus nécessaires ${ }^{91}$. Elle se livre ensuite à une lecture combinée de ces dispositions avec les articles 17.1 qui impose la protection de la famille et 11.2 qui protège toute personne contre les «ingérences arbitraires ou abusives [...] dans la vie de sa famille» en se fondant sur l'évolution du corpus juris international pertinent en la matière ${ }^{92}$. La Cour en déduit qu'en vertu de cette première disposition, l'État a l'obligation de garantir le maximum de contacts possible avec les membres de la famille des détenus, leurs représentants et le monde extérieur ${ }^{93}$. Bien qu'il ne s'agisse pas d'un droit absolu, différents facteurs doivent être pris en compte lors d'un transfert. Elle rappelle tout d'abord que «la peine doit avoir pour objectif principal la réadaptation ou la réinsertion du détenu». Ensuite,

[...] le contact avec la famille et le monde extérieur est essentiel à la réinsertion sociale des personnes privées de liberté [ce qui] implique le droit de recevoir des visites des membres de la famille et des représentants légaux.

Elle souligne dans un troisième temps que «la restriction des visites peut avoir un effet sur l'intégrité personnelle de la personne privée de liberté et de sa famille». Ensuite, "la séparation injustifiée des personnes privées de liberté de leur famille peut affecter l'article 17.1 de la Convention ainsi qu'éventuellement l'article 11.2». Enfin,

[...] dans le cas où le transfert n'a pas été demandé par la personne privée de liberté, elle doit être consultée, dans la mesure du possible, à chaque transfert d'une prison à une autre, et autorisée à s'opposer à ladite décision administrative, si besoin judiciairement ${ }^{94}$.

En l'espèce, la Cour passe en revue son test de compatibilité du transfert à l'article 5.6 de la Convention, à commencer par sa légalité. Au regard du pouvoir discrétionnaire laissé par la législation interne à l'administration pénitentiaire, elle estime que ce critère n'est pas rempli. Bien que cela suffise en principe à constater la violation de l'article 5.6, il lui paraît opportun de poursuivre son raisonnement au regard des quatre autres critères en vue de vérifier si l'ensemble des dispositions invoquées devant elle pouvaient l'être également ${ }^{95}$. S'agissant du but poursuivi avancé par l'État, à savoir la recherche «de meilleures conditions pour purger sa peine en vue de sa réinsertion, voire garantir sa sécurité », elle l'estime légitime ${ }^{96}$. La mesure est en revanche considérée comme ni adéquate, ni nécessaire, ni proportionnée ${ }^{97}$, d'où une

82. Ibid., $\$ 92$.

83. Cour IDH, Rodríguez Revolorio et autres $c$. Guatemala, $\$ 392$

84. Ibid.

85. La Cour était déjà parvenue à ce constat dans l'affaire Cour IDH, Fermín Ramírez c. Guatemala, $\$ 119$ et le réitère donc logiquement dans l'affaire Rodríguez Revolorio et autres c. Guatemala.

86. Cour IDH, Hernández c. Argentine, exception préliminaire, fond, réparations et frais, 22 novembre 2019 , série C, $\mathrm{n}^{\circ} 395$.

87. Ibid., $\$ 61$.

88. Voir l'analyse ci-dessous, au titre de l'article 26.

89. Cour IDH, López et autres c. Argentine, exceptions préliminaires, fond, réparations et frais, 25 novembre 2019, série $\mathrm{C}$, $\mathrm{n}^{\circ} 396$.

90. Il dispose que «[1]a peine est personnelle et ne peut frapper que le délinquant».

91. Selon la Cour, en effet: «Ce que vise l'article 5.3 est justement que les effets de la privation de liberté ne transcendent pas inutilement la personne du condamné, et ce au-delà de ce qui est indispensable» (Cour IDH, López et autres c. Argentine, \$ 93).

92. Cour IDH, López et autres c. Argentine, $\$ 90-117$.

93. Ibid., $\$ 118$.

94. Ces cinq facteurs sont énumérés dans le $\$ 118$ de cette même affaire.

95. Cour IDH, López et autres c. Argentine, $\$ 142$.

96. Ibid., $\$ 151$.

97. Ibid., \$ 152-158. 
violation des articles 5.1, 5.6, 11.2 et 17.1 à l'égard de la victime mais aussi à l'égard des membres de sa famille, la Cour soulignant en outre la particulière gravité de cette violation s'agissant de personnes mineures ${ }^{98}$.

\section{Le droit à la liberté de la personne (article 7 de la CADH)}

La Cour rappelle dans différentes affaires relatives à la détention préventive les critères qu'elle doit remplir pour être considérée comme compatible avec les articles 7.3, 7.5 et 8.2 de la Convention (lus de manière combinée). Ils sont listés dans l'affaire Romero Feris c. Argentine ${ }^{99}$. Pour qu' « une mesure conservatoire restreignant la liberté » ne soit pas considérée comme arbitraire, il faut:

i. que soient présentés des présupposés matériels se rapportant à l'existence d'un fait illicite et au lien entre la personne poursuivie et ce fait;

ii. que ces mesures remplissent les quatre autres critères du «test de proportionnalité», c'est-à-dire la finalité de la mesure qui doit être légitime (compatible avec la Convention américaine), son adéquation en vue d'atteindre le but recherché, sa nécessité et son caractère strictement proportionné, et

iii. que la décision qui l'impose contienne une motivation suffisante pour évaluer si elle remplit les conditions indiquées ${ }^{100}$

En l'espèce, la Cour revient sur le but poursuivi qu'est le déroulement efficace d'une procédure et la prévention d'un risque de fuite du suspect (considéré comme légitime) en soulignant que la gravité de la peine n'est pas, en soi, un critère suffisant pour le caractériser. L'imminence du jugement n'est pas non plus un argument. Enfin, le fait que la victime n'ait, selon le juge interne, pas reconnu la légitimité du tribunal qui le poursuivait et ne se soit pas prêté aux actes d'enquête n'est pas prouvé. La peine préventive est donc considérée comme arbitraire, d'où une violation conjuguée des articles 7.3, 7.5 et 8.2 de la Convention ${ }^{101}$.

\section{Les droits économiques, sociaux, culturels et environnementaux ${ }^{102}$ (article 26 de la CADH)}

Comme le constate Eduardo Ferrer Mac Greggor, la Cour fait la promotion depuis 2017 d'une vision globale des violations des droits humains soumis à son analyse, en insistant sur «l'interdépendance et l'indivisibilité des droits économiques, sociaux, culturels et environnementaux [...] et des droits civils et politiques ${ }^{103}$. En 2019, trois affaires sont particulièrement révélatrices de ce mouvement: les affaires Muelle Flores c. Pérou, ANCEJUB-SUNAT c. Pérou et Hernández c. Argentine. Dans les deux premières c'est le droit à une sécurité sociale et plus particulièrement le droit à une retraite qui est reconnu et, dans la troisième, le droit à la santé. La Cour adopte, dans ces trois affaires, une méthodologie similaire. Elle commence par affirmer ces droits comme droits autonomes et justiciables ${ }^{104}$, elle en fixe le contenu en se fondant sur un corpus juris international et national ${ }^{105}$ et elle termine en vérifiant si le droit en cause est violé en l'espèce ${ }^{106}$.

S'agissant du droit à une retraite, elle précise qu'il est une composante du droit à la sécurité sociale ${ }^{107}$. Il en découle plusieurs obligations à la charge des États, à savoir assurer le droit d'accéder à une retraite à l'âge légal prévu à cet effet qui suppose la mise en place d'un système de sécurité social efficace; garantir des prestations suffisantes en vue d'accéder à des conditions de vie adéquates; rendre effectifl'accès à une retraite; garantir qu'elle soit octroyée en temps utile et mettre en place un mécanisme judiciaire permettant d'invoquer une violation de ce droit ${ }^{108}$.

Le droit à la santé a quant à lui déjà été reconnu par la Cour dans plusieurs affaires antérieures, mais comme découlant de droits civils et politiques tels que le droit à la vie ou à l'intégrité ${ }^{109}$. L'affaire Hernández c. Argentine innove sur ce point puisque la Cour accepte de reconnaître qu'il a été directement violé, en tant que droit autonome et justiciable. Selon lui, l'État a le devoir d'assurer l'accès aux services de santé essentiels,

98. Cour IDH, López et autres c. Argentine, $\$ 159$.

99. Cour IDH, Romero Feris $c$. Argentine, fond, réparations et frais, 15 octobre 2019, série C, nº 391. Cette affaire concerne la détention préventive d'un élu ayant exercé différents mandats politiques entre les années 1985 et 1999, mis en cause pour des délits d'administration frauduleuse, d'enrichissement illicite, de malversation de fonds publics, d'abus de pouvoir, de fraude, entre autres.

100. Ibid., $\$$ 92. Voir aussi Cour IDH, Hernández c. Argentine, $\$ 103$ et Cour IDH, Jenkins c. Argentine, exceptions préliminaires, fond, réparations et frais, 26 novembre 2019 , série $C, n^{\circ} 397, \$ 74$.

101. Elle parvient à la même solution dans les affaires Cour IDH, Hernández c. Argentine, $\$ 100-117$ et Cour IDH, Jenkins c. Argentine, $\$ 72-82$.

102. Ci-après «DESCE». Notons que le qualificatif «environnementaux» est ajouté de manière prétorienne par la Cour notamment depuis son avis Cour IDH, Medio ambiente y derechos humanos (obligaciones estatales en relación con el medio ambiente en el marco de la protección y garantía de los derechos a la vida y a la integridad personal - interpretación y alcance de los artículos 4.1 y 5.1, en relación con los artículos 1.1 y 2 de la Convención Americana sobre Derechos Humanos), opinion consultative $\mathrm{n}^{\circ}$ OC-23/17, 15 novembre 2017, série A, $\mathrm{n}^{\circ} 23$. Sur ce point voir, inter alia, L. Burgorgue-Larsen, «Environnement et droits de l'homme: de l'audace du juge interaméricain des droits de l'homme», Énergie Environnement - Infrastructures, $\mathrm{n}^{\circ}$ 6, juin 2018, p. 53-55.

103. Eduardo Ferrer Mac Greggor, opinion consultative sous l’affaire Hernández c. Argentine, $₫ 1$.

104. Cour IDH, Muelle Flores c. Pérou, $\$$ 172-177; Cour IDH, ANCEJUB-SUNAT c. Pérou, \$156-161; et Cour IDH, Hernández c. Argentine, $\$ 64-67$.

105. Ibid., $\$ 178-193, \$ 162-176$ et $\$ 69-82$ respectivement.

106. Ibid., $\$ 194-207, \$ 177-191$ et $\$ 83-95$ respectivement.

107. Cour IDH, ANCEJUB-SUNAT c. Pérou, $\$ 184$

108. Ibid., $\$ 175$. La Cour précise en outre que les atteintes à ce droit par l'État affectent le droit à la propriété privée de leurs bénéficiaires (Cour IDH, Muelle Flores c. Pérou, $\$$ 212-217 et Cour IDH, ANCEJUB-SUNAT c. Pérou, \$192-195).

109. Voir sur ce point H. Tigroudja, «Chronique de la jurisprudence...», p. 704-707. 
garantissant des soins médicaux de qualité efficaces, mais aussi de promouvoir l'amélioration des conditions de santé de sa population ${ }^{110}$. D'après la Cour le droit à la santé se traduit par «le droit de toute personne de jouir du plus haut niveau de bien-être physique, mental et social» et il suppose que soient prodigués les soins «opportuns et appropriés conformément aux principes de disponibilité, d'accessibilité, d'acceptabilité et de qualité ${ }^{111}$. Enfin, l'État doit «accorder une attention particulière aux groupes vulnérables et marginalisés ${ }^{112}$.

Pour finir, la Cour revient dans ces trois affaires sur le caractère progressif des DESCE tel qu'il est mentionné dans l'article 26. S'agissant des deux droits dégagés, elle constate que «la nature et la portée des obligations qui [en] découlent» peuvent par certains aspects requérir « une exigibilité immédiate» et par d'autres « un caractère progressif ${ }^{113}$. Aussi, les États doivent tout d'abord «adopter des mesures efficaces en vue de garantir l'accès sans discrimination » à ces droits et, «d'une manière générale, progresser vers la pleine efficacité des DESCE»; ils ont, ensuite, «l'obligation concrète et constante de progresser aussi rapidement et efficacement que possible vers la pleine efficacité dudit droit, dans la mesure de leurs ressources disponibles, par le biais de la législation ou d'autres moyens appropriés " ${ }^{114}$. La Cour rappelle enfin le devoir de non-régression qui découle de l'article 26 tout en soulignant l'importance des articles 1.1 et 2 de la Convention pour parvenir à ces fins.

On peut voir en ces développements une victoire de l'ancien président de la Cour Eduardo Ferrer Mac Greggor, qui n'a eu de cesse de plaider en faveur de la reconnaissance de la justiciabilité directe des DESCE dans différentes opinions séparées depuis sa prise de fonction. La jurisprudence de l'année 2020, d'une Cour dorénavant présidée par Elizabeth Odio Benito, sera donc scrutée avec attention de ce point de vue.

110. Cour IDH, Hernández c. Argentine, $\$ 76-81$.

111. Ibid., $\$ 78$.

112. Ibid.

113. Cour IDH, Muelle Flores c. Pérou, \$190; Cour IDH, ANCEJUB-SUNAT c. Pérou, \$ 173; et Cour IDH, Hernández c. Argentine, $\$ 81$.

114. Ibid. 